\title{
AN EXTENSION OF HARTFIEL'S DETERMINANT INEQUALITY
}

\section{LEI Hou AND SHENG DONG}

Abstract. Let $A$ and $B$ be $n \times n$ positive definite matrices, Hartfiel obtained a lower bound for $\operatorname{det}(A+B)$. In this paper, we first extend his result to $\operatorname{det}(A+B+C)$, where $A, B$ and $C$ are $n \times n$ positive definite matrices, and then show a generalization of this to the case of matrices whose numerical ranges are contained in a sector.

Mathematics subject classification (2010): 15A45, 47A63.

Keywords and phrases: Hartfiel inequality, determinantal inequality, sector, numerical range.

\section{REFERENCES}

[1] S. DRURY, M. Lin, Singular value inequalities for matrices with numerical ranges in a sector, Oper. Matrices. 8, 4 (2014), 1143-1148.

[2] X. FU, Y. LIU, Rotfel'd inequality for partitioned matrices with numerical ranges in a sector, Linear Multilinear Algebra 64, 1 (2016), 105-109.

[3] A. George, K. H. D. Ikramov, On the properties of Accretive-Dissipative Matrices, Math. Notes. 77, 5-6 (2005), 767-776.

[4] D. J. HARTFIEL, An extension of Haynsworth's determinant inequality, Proc. Amer. Math. Soc. 41, 2 (1973), 463-465.

[5] E. V. Haynsworth, Applications of an inequality for the Schur complement, Proc. Amer. Math. Soc. 21, 3 (1970), 512-516.

[6] R. A. Horn, C. R. Johnson, Matrix Analysis, 2nd ed., Cambridge University Press, Cambridge, 2013.

[7] L. Hou, D. Zhang, Concave Functions of partitioned matrices with numerical ranges in a sector, Math. Inequal. Appl. 20, 2 (2017), 583-589.

[8] K. H. D. IKRAmov, Determinantal Inequalities for Accretive-Dissipative Matrices, J. Math. Sci. 121, 4 (2004), 2458-2464.

[9] M. Lin, Fischer type determinantal inequalities for accretive-dissipative matrices, Linear Algebra Appl. 438, 6 (2013), 2808-2812.

[10] M. LiN, A determinantal inequality for positive definite matrices, Electron J. Linear Algebra 27, 1 (2014), 821-826.

[11] M. Lin, Extension of a result of Hanynsworth and Hartfiel, Arch. Math. 104, 1 (2015), 93-100.

[12] M. Lin, D. ZHOU, Norm inequalities for accretive-dissipative operator matrices, J. Math. Anal. Appl. 407, 2 (2013), 436-442.

[13] J. LiU, Generalizations of the Brunn-Minkowski inequality, Linear Algebra and its Applications 508, 1 (2016), 206-213. 